\title{
Mixed tumour of the skin of the lower lip: A case report and review of the literature
}

\author{
SHIMPEI GOTOH ${ }^{1}$, EDWARD H. NTEGE ${ }^{1,2}$, TOSHIYUKI NAKASONE ${ }^{1}$, AKIRA MATAYOSHI $^{1}$, \\ SHO MIYAMOTO ${ }^{1}$, YUSUKE SHIMIZU ${ }^{2}$ and HIROYUKI NAKAMURA ${ }^{1}$ \\ Departments of ${ }^{1}$ Oral and Maxillofacial Surgery, and ${ }^{2}$ Plastic and Reconstructive Surgery, \\ Graduate School of Medicine, University of the Ryukyus, Nishihara, Okinawa 903-0215, Japan
}

Received July 20, 2021; Accepted December 16, 2021

DOI: $10.3892 / \mathrm{mco} .2022 .2502$

\begin{abstract}
Mixed tumour of the skin or chondroid syringoma (CS) is a rare and mostly benign neoplasm of the sweat glands. Although CS is frequently located on varied parts of the head and neck region, the lower lip is a rarely reported site. The present report describes a case of CS of the lower lip in a 58-year-old male as an expository case to further emphasise the need for proper diagnosis, appropriate treatment and prognostic evaluation. The patient presented with a round, non-tender, slightly hard and mobile mass beneath the mucocutaneous junction of his left lateral side of the lower lip. Radiology revealed a mass measuring $11 \times 11 \times 7 \mathrm{~mm}^{3}$ in size at a depth of $\sim 2 \mathrm{~mm}$. Furthermore, magnetic resonance T1- and T2-weighted images showed slightly low and high signal intensities, respectively. A provisional diagnosis of benign tumour of the lower lip was made, and surgical excision biopsy taken under local anaesthesia, while considering the patient's cosmetic appearance. Histopathology demonstrated features akin to apocrine gland, chondroid and myxoid stroma consistent with the diagnosis of benign CS. No evidence of recurrence or satellites were recorded after a follow-up of nearly 2 years. Although rare, a high index of suspicion for CS among other cutaneous adnexal tumours of the lower lip is necessary. In addition, interprofessional collaboration in the management of such oral tumours could enhance patient satisfaction amid prevailing intraoral and aesthetic concerns.
\end{abstract}

Correspondence to: Professor Hiroyuki Nakamura, Department of Oral and Maxillofacial Surgery, Graduate School of Medicine, University of the Ryukyus, 207 Uehara, Nakagami, Nishihara, Okinawa 903-0215, Japan

E-mail: hnak@me.com

Abbreviations: CK, cytokeratin; ECD, extracapsular dissection; EMA, epithelial membrane antigen; HMGA2, high-mobility group AT-hook 2; p63, tumor protein 63 aka transformation-related protein 63; PLAG1, pleomorphic adenoma gene 1; SMA, smooth muscle actin protein; US, ultrasonography

Key words: chondroid syringoma, mixed tumour of the skin, lower lip, cutaneuos adnexal tumour, oral cavity

\section{Introduction}

Mixed tumour of the skin also known as chondroid syringoma (CS) is a rare, mostly benign tumour categorized under cutaneous adnexal neoplasms. These neoplasms exhibit morphologic differentiation towards the primary adnexal structures found in normal skin, including hair follicles, sebaceous glands and apocrine and eccrine (sweat) glands (1). CS was first described by Billroth in 1859 as a tumor on the salivary glands, a few years later, Virchow regarded the tumour as being identical to mesenchymal neoplasm and called it 'mixed tumor'. However, the first case of mixed tumor of the skin was reported by Nasse in 1892 (2,3). Several decades later in 1961, upon a review of 188 cases of mixed tumour of the skin, the name 'Chondroid syringoma' was coined (4). Although contested, the Hirsch and Helwig (4) chondroid terminology resulted from observation that the biggest number of mostly scalp lesion cases had cartilaginous properties in the tumor stroma $(4,5)$. Other tumour locations, reportedly demonstrate a myxoid stroma (5). Moreover, several mixed tumour studies by Headington (6) and Hassab-el-Naby et al (7) have demonstrated 2 morphologically distinct types of mixed tumours referred to as apocrine-type cutaneous mixed tumor (AMT) and eccrine-type cutaneous mixed tumor (EMT).

The incidence of CS is reportedly $<0.36 \%$ of all primary skin tumours (2). The tumour affects mostly middle-aged men, and commonly found in various sites of the head and neck region including the scalp, nose, cheek and lips (2-4). However, from professional literature more cases in the upper lips have been described compared to the lower lips (5-26).

The clinical diagnosis of CS is mainly based on pathology that demonstrates the epithelial and mesenchymal components of CS. Histology shows the tubular and glandular structures with one or two layers of epithelial cells-inner and outer cells, in a fibrous or chondroid stroma (matrix). However, the presence of minor salivary glands in the lower lip poses a challenging CS diagnostic problem. Cutaneous adnexal tumours, such as pleomorphic adenoma (PA) of the salivary gland exhibit similar clinical and histopathological features and may localise in the lower lip as well (27-29). Further, there is a strong possibility of a close molecular level relationship between CS and PA tumor types (30). The genetic or protein expression profile of the 2 tumour types is not yet completely deciphered. 
However, recent work has demonstrated that both tumours possess similar chromosomal rearrangements involving genes such as the fusion genes encoding pleomorphic adenoma gene 1 (PLAG1) zinc finger protein and the high-mobility group AT-hook 2 (HMGA2) protein $(30,31)$. Notwithstanding, Russell-Goldman et al (31) have recently demonstrated that the mechanisms of expression of these genes could be different for the AMT and EMT tumour types and suggested the molecular relationship between CS and PA maybe limited to AMT tumour type. Additionally, the immunohistochemistry (IHC) of CS reveals a different protein marker expression profile for each of the three cellular components-inner, outer and matrix. The inner component expression profile includes inter alia cytokeratins (CK), carcinoembryonic antigen (CEA) and epithelial membrane antigen (EMA). However, the outer and matrix expression profile includes vimentin, S100 protein, neuron-specific enolase, glial fibrillary acidic protein or different types of actin, such as the smooth muscle actin protein (SMA) (27,32).

The objective of this report is to document this rare benign skin lesion of the lower lip in a 58-year-old man, and promote clinical awareness, recapitulate the diagnostic challenges and emphasise the importance of appropriate treatment and prognostic evaluation.

\section{Case report}

Patient description. A 58-year-old Japanese man was referred to the Department of Oral and Maxillofacial Surgery at the University of the Ryukyus Hospital by a private practitioner in October 2019. The patient complained of a slow-progressing, painless swelling in the lower lip, which followed an accidental biting of the inside of the lip in 2010. In addition, the patient had fears of anecdote postoperative functional and aesthetic effects of surgery.

Physical examination revealed the patient was in good general condition, with no pallor, oedema, jaundice or cervical or generalised lymphadenopathy; and systemic examinations were within normal limits. Intraoral examination was unremarkable; however, a non-tender, slightly hard and mobile mass measuring $\sim 11 \times 11 \times 7 \mathrm{~mm}^{3}$ in size was felt on palpating the left lateral side of the lower lip (Fig. 1). The overlying mucocutaneous surface appeared normal and smooth without evidence of ulceration.

Investigations. With differential diagnoses of epidermal or dermoid cyst, neurofibroma and dermatofibroma, the patient underwent radiological, routine haematological and pathological investigations. Radiology involved ultrasonography (US) and magnetic resonance imaging (MRI) of the lip and oral cavity respectively. US was performed with an $18-\mathrm{MHz}$ scanner and a L64 linear transducer or probe (Hitachi Aloka Medical, Ltd.). The probe was fitted with a rubber sheath and filled with degassed water to minimize acoustic artifacts and ensure acoustic coupling. It was used without the water-soluble gel. The examination was performed with the patient opening the mouth, and gently extending the lower lip with gauze. Light pressure was applied to the surface of the lesion to prevent shape distortion. MRI was performed using a 3.0-T Ingenia Elition X Philips system (Philips Healthcare) scanner. Briefly, the patient underwent 3-dimensional (3D) spoiled-gradient multiple echo modified Dixon (mDIXON) sequence on the scanner using the following acquisition parameters: 2 echoes ( $\mathrm{n} \times 1.94 \mathrm{~ms}$ echo time (TE) with $\mathrm{n}=3.3$ ), $15^{\circ}$ flip angle, $6.0 \mathrm{msec}$

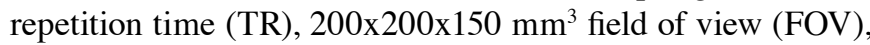
$224 \times 224 \times 150$ matrix size, $3 \mathrm{~min} 9 \mathrm{sec}$ scan time. The acquisition was in single breath-hold with patient in supine position. For signal reception, a 20 channel body-array coil was used. The acquisition was performed on the Head and Neck region.

Histopathological evaluations utilised fresh surgical excision biopsy obtained by extracapsular dissection (ECD) of the lower lip lesion. Briefly, after finding the haematology results within normal ranges, the excisional biopsy was performed over the intraoral side of the lower lip mucocutaneous junction, through a $15 \mathrm{~mm}$ vertical incision in the lateral third of the lip. The tumor was well circumscribed with no adhesions and was easily bluntly dissected and removed in its entirety including a free margin of about $3 \mathrm{~mm}$. The incision was closed with a 6.0 polypropylene suture and the entire specimen was submitted to the university hospital pathology department for histologic examination. In the department, standard diagnostic laboratory histopathological staining protocols and interpretation of obtained images were observed. Hematoxylin and eosin (H\&E) staining was done on $96 \%$ ethanol fixed frozen biopsy material cut into $4 \mu \mathrm{m}$ thick sections. The fixed sliced sections-slides, were incubated in a $10 \%$ formalin solution for $24 \mathrm{~h}$. Thereafter, slides were rinsed with sterile water before staining with hematoxylin for $30 \mathrm{sec}$. After hematoxylin staining, the slides were rinsed under running water for $5 \mathrm{~min}$, before counterstaining with eosin Y for $15 \mathrm{sec}$. Finally, the slides were dehydrated in ethanol (96 and 99.8\%), fixed with xylene, and coverslipped with a mounting medium. All the diagnostic images were taken under a Nikon Eclipse CI microscope and captured with a Nikon DS-Fi3 camera. (Nikon Eclipse Ci). Differential staining of acid mucopolysaccharides by AIcian blue was achieved by staining the fixed slides with $1 \%(\mathrm{w} / \mathrm{v})$ Alcian Blue $8 \mathrm{GX}$ (Sigma) in $3 \%(\mathrm{v} / \mathrm{v})$ acetic acid (pH 2.5). Then microwaved for $5 \mathrm{~min}$ at $80 \mathrm{~W}$, followed by an additional 10-min incubation in the warm Alcian blue solution, and then washed three times in distilled water, dehydrated and mounted with dibutyl phathalate xylene (DPX) Mountant (Sigma-Aldrich; cat. no. 44581) before the microscopy examination (Nikon Eclipse Ci).

IHC utilised the streptavidin-peroxidase technique on biopsy sections of $4-\mu \mathrm{m}$ thickness. Briefly, the $4-\mu \mathrm{m}$ sections were rinsed with phosphate-buffered saline (PBS) and pepsase activity of $10 \mathrm{~min}$ was used for antigen retrieval. After washing in PBS, the sections were incubated in methanol with addition of $3 \%$ hydrogen peroxide to deactivate the endogenous peroxidases and blocked with PBS 0.5\% Tween-20 (PBS-T; Sigma), containing 3\% bovine serum albumin (BSA) (Sigma) for $1 \mathrm{~h}$ at room temperature. The slides were incubated at $4^{\circ} \mathrm{C}$ overnight with primary antibodies of epithelial markers including CK7, CK15, EMA, and CEA and mesenchymal and myoepithelial markers including SMA, S-100, and tumor protein 63 aka transformation-related protein 63 (p63). For instance, for the basal epithelial differentiation marker CK15, the primary antibody, rabbit anti CK15 (1:200; ab52816; Abcam) was used. After three washes with PBS, a secondary antibody, goat anti-rabbit IgG H\&L (HRP) (1:1,000; ab205718; Abcam) was 


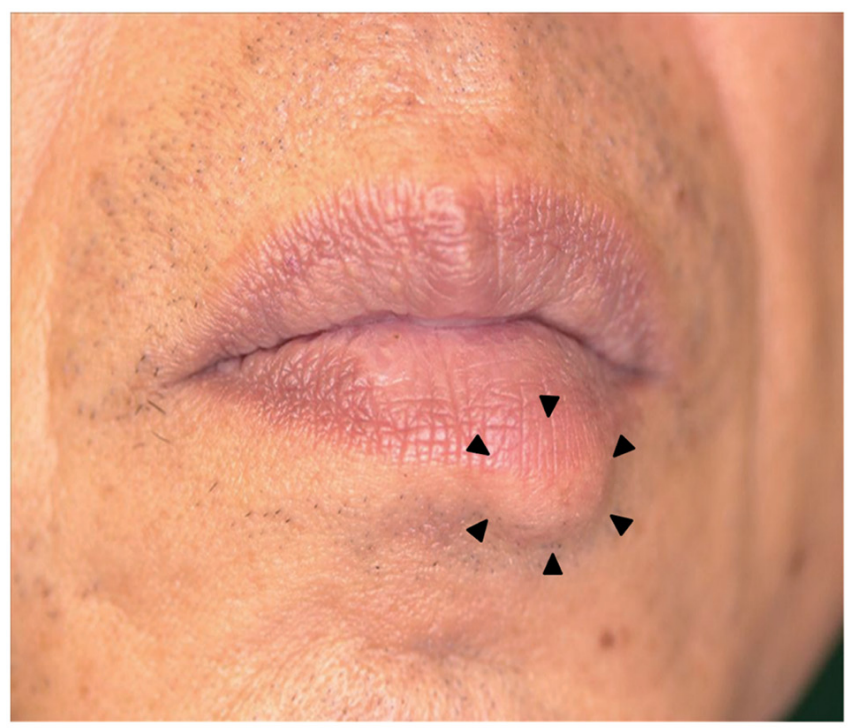

Figure 1. Photograph of the 58-year-old male patient. Arrowheads (black) indicate the location of the tumour in the left lower lip.

added with fresh Diaminobenzidine as the substrate. Negative controls utilized PBS as a replacement for the primary antibody. All images were acquired using Philips IntelliSite Pathology Solution (Philips), and analysed by at least 2 pathologists.

Results. US revealed a well-defined, heterogenous, hypoechoic lesion measuring $11 \times 11 \times 7 \mathrm{~mm}^{3}$ in size at a depth of $\sim 2 \mathrm{~mm}$ in subcutaneous plane of the lower lip with increased internal vascularity, but no lymph node swelling or other tumourous lesions were identified (Fig. 2A). MRI demonstrated low signal intensity on $\mathrm{T} 1$ weighted images and intermediate to high signal intensity on T2 weighted images in the left lateral third of the lower lip (Fig. 2B and C). ECD revealed a tumour covered with a capsula fibrosa located between the orbicularis oris muscle and the skin (Fig. 3). On gross examination, the excision biopsy was round, yellowish-white tumour covered by a thin fibrous capsule (Fig. 4).

Histologically, a relatively well-defined nodular lesion was observed (Fig. 5A) with features ranging from mucinous interstitium, proliferation and migration of short-spindle stellate cells that comprised epithelial nests and many cysts mimicking fused and scattered epithelial and hair follicle infundibula and partially differentiated bone, cartilage and adipose tissue structures. In addition, IHC showed inner epithelial cell expression of CK15, CK7, EMA, and CEA and outer epithelial cells expression of mesenchymal and myoepithelial markers, S-100, SMA and p63. However, the expression of CK15 (Fig. 5B) and p63 were stronger (higher) compared to the other markers (data not shown). A diagnosis of CS was retrospectively made.

Treatment and follow-up. The ECD surgery performed to obtain a complete free margin excision biopsy also served as the definitive treatment for the benign CS. Moreover, the patient was offered preoperative counselling to reduce anxiety and enhance postoperative satisfaction through interprofessional collaboration practice with the plastic surgery team. The patient had an uneventful healing (Fig. S1) and the MRI
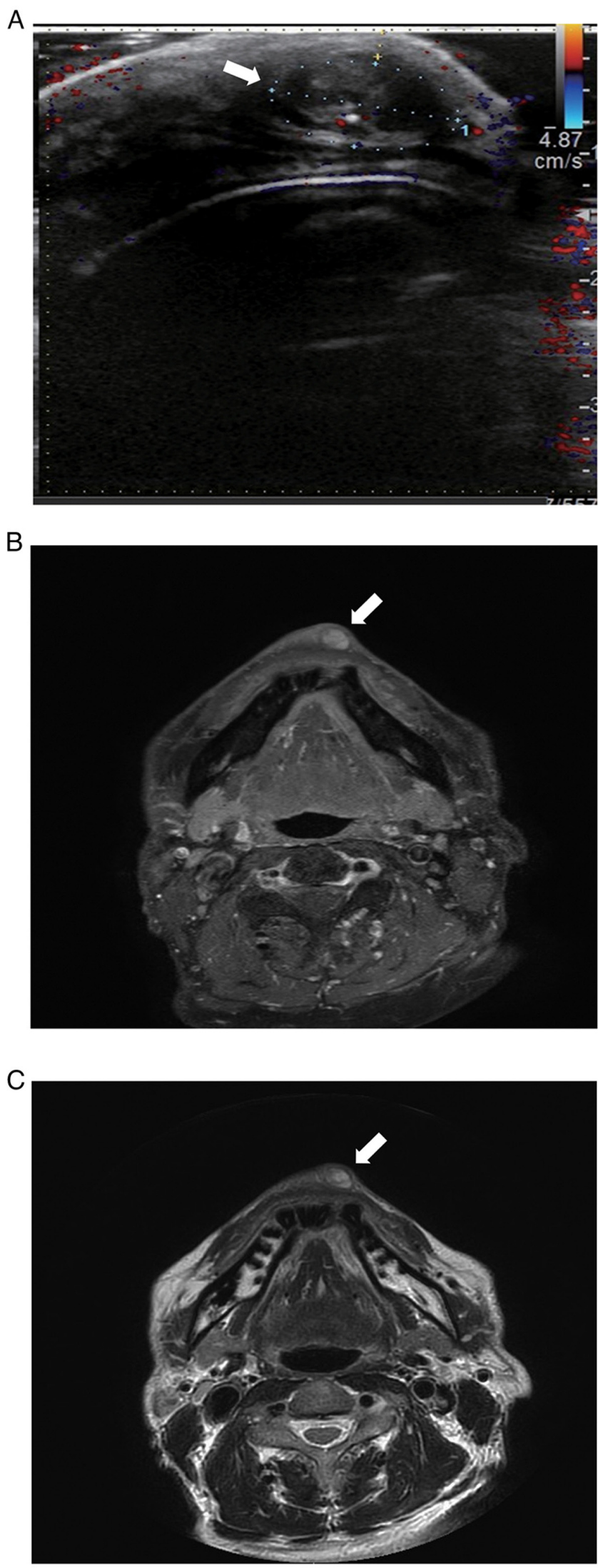

Figure 2. Preoperative US and MRI examination of the tumour. (A) Ultrasonogram showing a heterogeneously enhanced, $7 \mathrm{~mm}$ round mass in the left lateral side of the lower lip (white arrow). (B) MRI T1-weighted image showing the lesion with low signal intensity in the left lateral side of the lower lip (white arrow). (C) MRI T2-weighted image showing a well-defined mass of high signal intensity in the left lateral side of the lower lip (white arrow). US, ultrasonography; MRI, magnetic resonance imaging. 


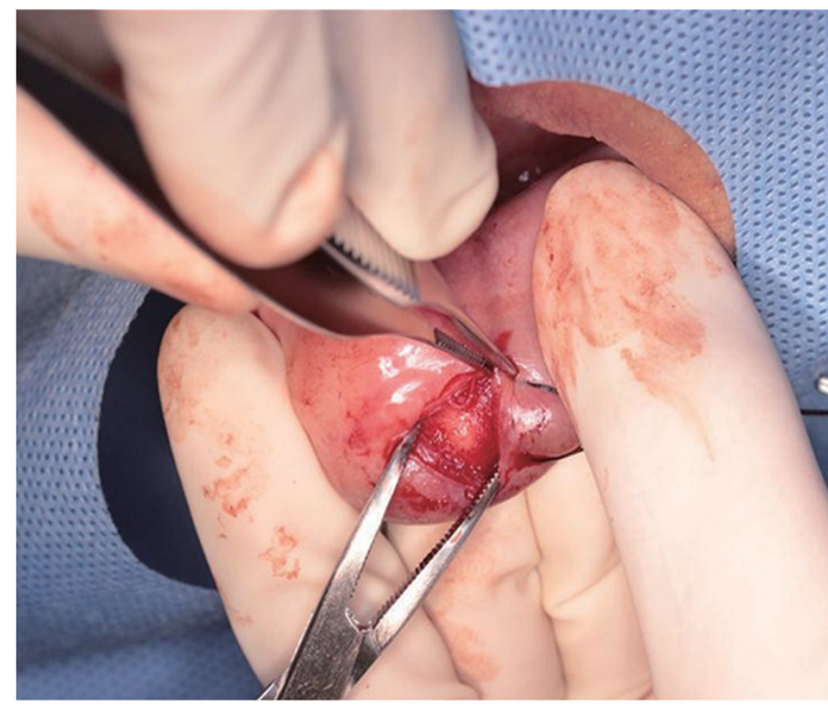

Figure 3. Surgical excision of the tumour. The tumour was covered with a capsula fibrosa and was exposed between the orbicularis oris muscle and the skin.

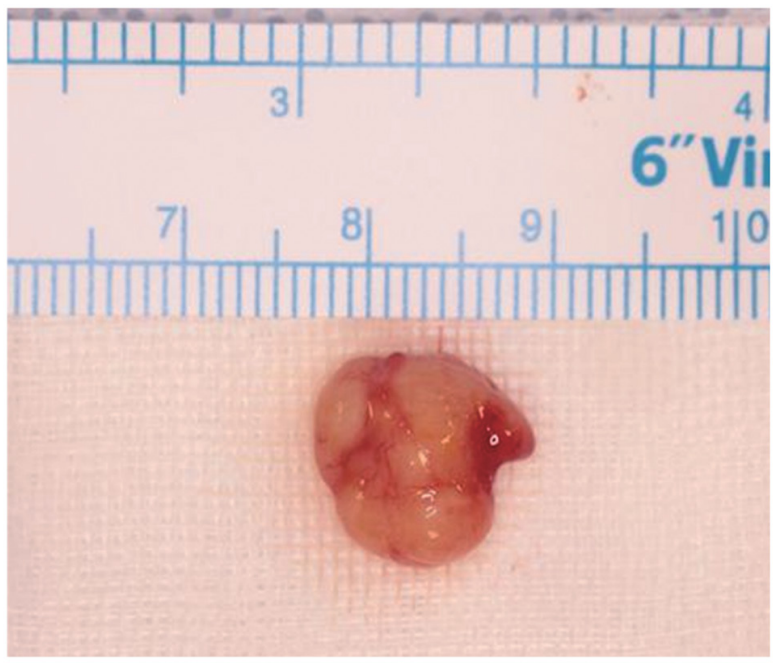

Figure 4. Complete excision biopsy of the tumour. The resected material was a round yellowish-white mass measuring $\sim 7 \times 11 \times 6 \mathrm{~mm}^{3}$ in size and was covered by a thin capsula fibrosa.

after a follow-up of nearly 2 years did not indicate evidence of recurrence (Fig. S2).

\section{Literature review}

Literature search strategy. The literature search for review, summarized in a schema (Fig. S3), mainly focused on original articles about patients with cutaneous mixed tumours of the head and neck region that were written in English. Non-English articles were evaluated for pertinence and content of new information. Databases including inter alia Medline, Embase and Web of Science were searched using various combinations of the following search terms: Head and Neck region, cutaneous adnexal tumours, mixed tumour of the skin, chondroid syringoma, upper lip, and lower lip. There was no restriction on date of publication and the earliest article used was published in 1859. However, the review excluded articles
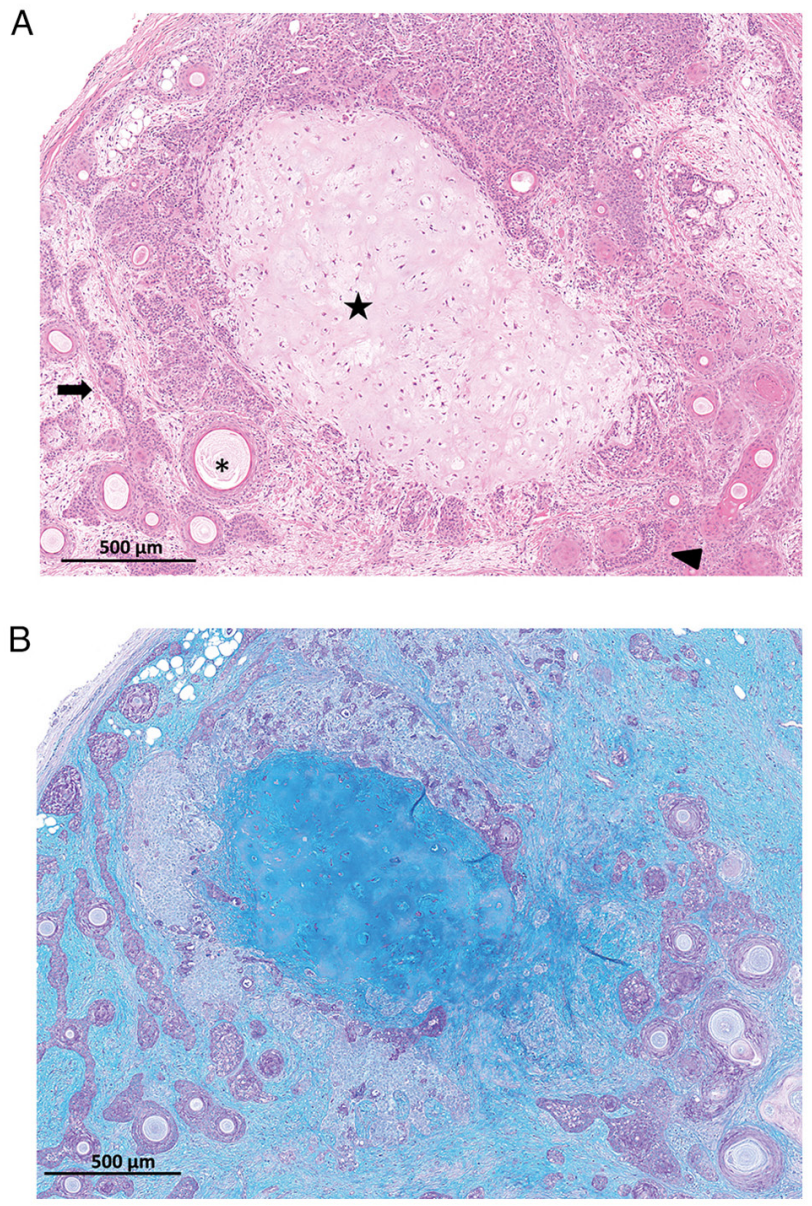

Figure 5. Histological examination of the biopsy material. (A) Hematoxylin and eosin (H\&E) staining showing myxoid stroma with the tumour growing in the shape of an aster (arrowhead) or cord (arrow), partial cartilage components (star) and apocrine gland differentiation (asterisk). Magnification, x40. (B) Alcian blue staining with acid mucopolysaccharide in cartilage and fibrous connective tissue. Immunostaining. Magnification, $x 40$.

of other mixed tumours, malignant tumours, salivary gland tumours, pleomorphic adenoma, or the benign cutaneous mixed tumours located on other parts of the body including trunk and extremities.

Articles were screened manually and selected according to the inclusion and exclusion criteria in a two-step process by two authors (SG and EHN). Selected articles were reviewed based on the title and abstract before screening the full texts.

Findings. The literature search resulted in the identification of a total of 394 related records (Fig. S3). Of these, 336 entries for other tumours, such as PA and malignant tumours were excluded. Based on the title and abstract review, 38 records of CS localized in other parts of the body were dropped, leaving 20 articles eligible for full-text review. The full-text review is summarized in Table I where 17/20 (85\%) articles reported 38 cases of CS of the upper lip and only 3/20 (15\%) articles reported 4 cases of CS of the lower lip (Table I).

\section{Discussion}

Mixed tumour of the skin, arguably referred to as CS, is a rare and mostly benign skin lesion, comprising both epithelial and 
Table I. Published cases of chondroid syringoma in the lip.

\begin{tabular}{|c|c|c|c|c|c|}
\hline Authors, year & Lip location & Number of patients & Sex & Age, years & (Refs.) \\
\hline Stout and Gorman, 1959 & \multirow[t]{16}{*}{ Upper lip $(n=38)$} & 16 & - & - & $(10)$ \\
\hline Triantafyllou and Rapidis, 1986 & & 1 & Male & 38 & (12) \\
\hline Adlam and Wood, 1986 & & 1 & - & - & (14) \\
\hline Zumdick et al, 1995 & & 1 & Male & 85 & (24) \\
\hline Shimizu et al, 1996 & & 1 & Female & 68 & $(25)$ \\
\hline \multirow[t]{2}{*}{ Bekerecioglu et al, 2002} & & 3 & Male & 27 & (9) \\
\hline & & & Females & 24,28 & \\
\hline Satter and Graham, 2003 & & 1 & Male & 25 & $(26)$ \\
\hline Shimizu et al, 2003 & & 1 & Male & 68 & (16) \\
\hline Arikan et al, 2004 & & 1 & Male & 73 & $(15)$ \\
\hline Dubb and Michelow, 2010 & & 1 & Female & 58 & (17) \\
\hline Girgis et al, 2015 & & 1 & Male & 23 & (18) \\
\hline Shilpa et al, 2016 & & 1 & Male & 48 & (11) \\
\hline Kundu et al, 2016 & & 1 & Male & 46 & (19) \\
\hline \multirow[t]{2}{*}{ Min et al, 2016} & & 5 & Males & $39,44,47,64$ & $(20)$ \\
\hline & & & Female & 65 & \\
\hline Reddy et al, 2018 & & 1 & Male & 35 & (13) \\
\hline Syed et al, 2019 & & 1 & Male & 44 & $(22)$ \\
\hline Vázquez Hernández et al, 2021 & & 1 & Male & 65 & $(21)$ \\
\hline Stout and Gorman, 1959 & Lower lip & 2 & - & - & $(10)$ \\
\hline Rodrigues et al, 2021 & $(n=4$ in searched & 1 & Male & 43 & (8) \\
\hline Palit et al, 2021 & articles) & 1 & Male & 42 & $(23)$ \\
\hline Present study & & 1 & Male & 58 & - \\
\hline
\end{tabular}

mesenchymal stromal derived elements $(4,5)$. CS can originate either from apocrine or eccrine sweat glands $(6,7)$ or result from idiopathic proliferation of abnormally located embryonic tissue (33). As observed in our patient medical history, it is also suggested that trauma could be a predisposing factor to the development of CS $(8,33)$.

The most common CS site location in the head and neck region is the face, especially on the nose and its surroundings (4). Hirsch and Helwig (4) reported a 79.8\% occurrence of CS in the head or maxillofacial region and highlighted the lower lip site as extremely rare, with only 2 out of 150 cases $(1.3 \%)$. Similarly, Stout and Gorman (10) reported only 2 out of 134 cases $(1.5 \%)$ of $\mathrm{CS}$ in the head and neck region occurred on the lower lip. Consistent with previous reports $(4,10)$, our professional literature search indicated that CS of the lower lip remains rare with only 4 out of 42 cases $(9.5 \%)$ reported in the identified articles on CS of the lips (8-26).

CS mostly manifests clinically as a painless, isolated, solid, elastic and hard mass, with a relatively slow increase in size and an average diameter of $\sim 1 \mathrm{~cm}(12,34,35)$. CS has no specific clinically observable characteristics (36). Hence, histopathological examinations are indispensable in distinguishing CS from other mixed tumours of skin, such as the PA. Histologically, the tumour with ducts surrounded by a bilayer of epithelium and forms a dilated lumen or cystic cavity is the AMT type, whereas the one that forms a small lumen surrounded by a single layer of epithelium is the EMT type (37). The sweat glands found on facial skin are heteroge- neous, and comprise both eccrine and apocrine glands. The eccrine glands are distributed all over the facial skin except the lips, whereas the apocrine glands are mainly distributed on the alae nasi, nasal vestibule and ear canal. Notably, although the beard area of the face contains apocrine glands (38), they are comparatively less distributed in the lower lip. This might justify the reported low incidence of CS in this site. In our case, morphological differentiation toward the hair follicles was illustrated. Further, although IHC markers are of limited value to differentiate AMT from EMT types of CS, the findings of especially the strong expression of CK15 and p63 together with EMA and CEA suggested the tumour to be of AMT type. CEA is positive on the secretory lumen of secretory cells and the luminal surface of the duct in eccrine sweat glands, while on the apocrine secretory cells, it may also label the luminal membranes (34). The stromal component in CS may be myxoid, chondroid, adipocytic or fibrous. In our case, the stromal cells showed myoepithelial differentiation as suggested by the positive expression of S100 protein. However, most important diagnostic findings were the illustration of histomorphology of CS including the differentiation toward bone, chondroid and adipose tissue in this case. Histology did not show features suspicious of malignancy such as cytological atypia, increased mitotic figures, infiltrative margins, satellite tumor nodules or tumor necrosis. In addition, there was no evidence of recurrence or metastases after a follow-up period of nearly 2 years. Hence, a diagnosis of atypical or malignant CS was excluded. 
In principle, the treatment of CS is complete surgical excision (5). Inadequate surgical excision of CS reportedly causes recurrence (39) and malignant transformation $(40,41)$. Therefore, a careful determination of the extent of resection should always be done; and ensure a regular postoperative examination and long-term follow-up of patients $(40,41)$. In this case, because the patient had aesthetic concerns, we opted for the less invasive and safer ECD surgical approach commonly subjected to surgery of the parotid gland. Additionally, we approached the excision of the tumour from the intraoral side to limit exposure of the surgical scar. Interprofessional collaborative practice of managing the tumour with the plastic surgery team allayed the patient's anxieties and positively influenced postoperative satisfaction.

In conclusion, although CS of the lower lip is a rare tumor, it should be considered in the differential diagnosis of lip masses. Interprofessional collaborative management of the patients is encouraged where necessary and should include complete excision of the lesion and long-term follow-up since malignant forms of CS have been reported.

\section{Acknowledgements}

Not applicable.

\section{Funding}

No funding was received.

\section{Availability of data and materials}

The datasets used and/or analysed during the current study are available from the corresponding author on reasonable request.

\section{Authors' contributions}

SG, EHN, YS and HN were involved in the conception and design of the report. SG, EHN, TN, AM and SM analysed and interpreted the patient data. SG, EHN and AM drafted and critically revised the manuscript for important intellectual content. YS and HN confirmed the authenticity of all the raw data and provided final approval of the completed article. All authors have read and approved the final manuscript.

\section{Ethics approval and consent to participate}

Not applicable.

\section{Patient consent for publication}

Written informed consent was obtained from the patient for both the surgical treatment and publication of any accompanying images.

\section{Competing interests}

The authors declare that they have no competing interests.

\section{References}

1. Sulochana S, Manoharan M and Anith: Chondroid syringoma-an unusual presentation. J Clin Diagn Res 8: FD13-FD14, 2014.

2. Billroth T: Observations on the tumors of salivary glands. Archiv Pathol Anat Physiol Clin Med 17: 357-375, 1859.

3. Gianotti R, Coggi A and Alessi E: Cutaneous apocrine mixed tumor: Derived from the apocrine duct of the folliculo-sebaceous-apocrine unit? Am J Dermatopathol 20: 53-55, 1998.

4. Hirsch $\mathrm{P}$ and Helwig EB: Chondroid syringoma. Mixed tumor of skin, salivary gland type. Arch Dermatol 84: 835-847, 1961.

5. Bergeron S, Ito $\mathrm{H}$, Arthurs $\mathrm{B}$ and Burnier MN Jr: Pleomorphic adenoma of the eyelid skin: A series of three atypical cases. Human pathology: Case Reports 20: 200384, 2020.

6. Headington JT: Mixed tumors of skin: Eccrine and apocrine types. Arch Dermatol 84: 989-996, 1961.

7. Hassab-el-Naby HM, Tam S, White WL and Ackerman AB: Mixed tumors of the skin. A histological and immunohistochemical study. Am J Dermatopathol 11: 413-428, 1989.

8. Rodrigues BTG, Romañach MJ, de Andrade BAB, de Almeida Freire N and Israel MS: Chondroid syringoma of the lower lip: Case report. J Oral Maxillofac Surg Med Pathol 33: 486-488, 2021.

9. Bekerecioglu M., Tercan M, Karakok M, and Atik B. Benign chondroid syringoma: a confusing clinical diagnosis. Eur J Plast Surg 25: 316-318, 2002.

10. Stout AP and Gorman JG: Mixed tumors of the skin of the salivary gland type. Cancer 12: 537-543, 1959.

11. Shilpa K, Leelavathy B, Divya G and Lakshmi D: Chondroid syringoma: Histopathology a cornerstone tool in diagnosis. Indian J Dermatopathol Diagn Dermatol 3: 20, 2016.

12. Triantafyllou AG and Rapidis AD: Chondroid syringoma of the upper lip: Report of a case. J Oral Maxillofac Surg 44: 744-748, 1986.

13. Reddy PB, Nandini DB, Sreedevi R and Deepak BS: Benign chondroid syringoma affecting the upper lip: Report of a rare case and review of literature. J Oral Maxillofac Pathol 22: 401-405, 2018.

14. Adlam DM and Wood GA: The chondroid syringoma (mixed tumor of skin). Report of a case in the upper lip. Oral Surg Oral Med Oral Pathol 61: 69-72, 1986.

15. Arikan OK, Erdoğan S, Muluk NB and Koç C: Chondroid syringoma of the upper lip: A case report. Kulak Burun Bogaz Ihtis Derg 13: 25-27, 2004.

16. Shimizu M, Kawano K, Fujiwara S, Noguchi T and Goto Y: Mixed tumor of the skin (chondroid syringoma) occurring in the upper lip: Report of a case. J Jpn Soc Oral Oncol 15: 37-41, 2003 (In Japanese).

17. Dubb M and Michelow P: Cytologic features of chondroid syringoma in fine needle aspiration biopsies: A report of 3 cases. Acta Cytol 54: 183-186, 2010.

18. Girgis S, Gillan G and Piper K: Rare benign mixed tumour of the upper lip: A case report. Ann Med Surg (Lond) 4: 380-383, 2015.

19. Kundu R, Punia RS, Handa U and Dalal U: Chondroid syringoma: Cytomorphology of four cases and review of literature. Arch Cytol Histopathol Res 1: 63-67, 2016

20. Min KH, Byun JH, Lim JS, Lee HK, Lee WM and Joo JE: Chondroid syringoma on face. Arch Craniofac Surg 17: 173-175, 2016.

21. Vázquez Hernández A, Pérez Campos AE, Gamboa Jiménez TI and Fenton Navarro BF: Giant chondroid syringoma on the upper lip: A case report. Dermatol Online J: May 15, 2021 (Epub ahead of print). doi: 10.5070/D327553622, 2021.

22. Syed MA, Paudel U, Rajbhandari A, Pokhrel DB, Adhikari RC and Parajuli S: Fine needle aspiration cytology as a preliminary diagnostic tool in chondroid syringoma: A case report and review. Clin Cosmet Invest Dermatol 12: 209-218, 2019.

23. Palit A, Sethy M, Nayak AK, Ayyanar P and Behera B: Dermoscopic features in a case of chondroid syringoma. Indian J Dermatol Venereol Leprol 87: 89-92, 2021.

24. Zumdick M, Milde P, Ruzicka T and Hölzle E: Apokriner kutaner Mischtumor mit follikulärer Differenzierung. Der Hautarzt 46: 481-484, 1995.

25. Shimizu S, Han-Yaku H, Fukushima S and Shimizu H: Immunohistochemical study of mixed tumor of the skin with marked ossification. Dermatology 193: 255-257, 1996.

26. Satter EK and Graham BS: Chondroid syringoma. Cutis 71: 49-55, 2003. 
27. Wan $\mathrm{H}, \mathrm{Xu} \mathrm{M}$ and Xia T: Clinical and pathological study on mixed tumors of the skin. Medicine (Baltimore) 97: e12216, 2018

28. Greeley PW, Gleason MC and Curtin JW: Mixed cell tumors of the skin. Plast Reconstr Surg (1946) 18: 427-435, 1956.

29. Chęciński M, Sikora M, Sielski M and Chlubek D: Pleomorphic adenoma of the lip-a case report. Pomeranian J Life Sci 67: 27-32, 2021

30. Matsuyama A, Hisaoka M and Hashimoto H: PLAG1 expression in cutaneous mixed tumors: An immunohistochemical and molecular genetic study. Virchows Arch 459: 539-545, 2011.

31. Russell-Goldman E, Dubuc A and Hanna J: Differential expression of PLAG1 in apocrine and eccrine cutaneous mixed tumors: Evidence for distinct molecular pathogenesis. Am J Dermatopathol 42: 251-257, 2020.

32. Nakayama H, Miyazaki E, Hiroi M, Kiyoku H, Naruse K and Enzan H: So $\square$ called neoplastic myoepithelial cells in chondroid syringomas/mixed tumors of the skin: Their subtypes and immunohistochemical analysis. Pathol Int 48: 245-253, 1998

33. Paik YS and Liess BD: Chondroid syringoma of the scalp: Case report and discussion of clinical features, histopathology, and treatment. Ear Nose Throat J 90: 190-191, 2011.

34. Saga K: Histochemical and immunohistochemical markers for human eccrine and apocrine sweat glands: An aid for histopathologic differentiation of sweat gland tumors. J Investig Dermatol Symp Proc 6: 49-53, 2001.

35. Kakuta M, Tsuboi R, Yamazaki M, Sakuma M, Yoshikata R and Ogawa H: Giant mixed tumor of the face. J Dermatol 23: 369-371, 1996
36. Kerimoglu U, Aydingoz U, Ozkaya O, Aksu AE and Ergen FB: MRI of a benign chondroid syringoma. Br J Radiol 79: e59-e61, 2006.

37. Kunikane H, Ishikura H, Yamaguchi J, Yoshiki T, Itoh T and Aizawa M: Chondroid syringoma (mixed tumor of the skin). A clinicopathological study of 13 cases. Acta Pathol Jpn 37: 615-625, 1987.

38. Ishimura E, Iwamoto H, Kobashi Y, Yamabe H and Ichijima K: Malignant chondroid syringoma. Report of a case with widespread metastasis and review of pertinent literature. Cancer 52: 1966-1973, 1983.

39. Sharvill DE: Mixed salivary-type tumour of the skin with malignant recurrence. Br J Dermatol 74: 103-104, 1962.

40. Redono C, Rocamora A, Villoria F and Garcia M: Malignant mixed tumor of the skin: Malignant chondroid syringoma. Cancer 49: 1690-1696, 1982.

41. Gardner JM and Smoller BR: Malignant mixed tumor of skin. In: Rare Malignant Skin Tumors. Springer, New York, NY, pp77-80, 2015.

This work is licensed under a Creative Commons Attribution-NonCommercial-NoDerivatives 4.0 International (CC BY-NC-ND 4.0) License. 